\title{
Growth Diagrams for Grip Strength in Children
}

\author{
H. M. (Ties) Molenaar MSc, Ruud W. Selles PhD, J. Michiel Zuidam MSc, \\ Sten P. Willemsen MSc, Henk J. Stam, Steven E. R. Hovius
}

Received: 28 November 2008/ Accepted: 24 April 2009/Published online: 21 May 2009

(C) The Author(s) 2009. This article is published with open access at Springerlink.com

\begin{abstract}
Grip strength dynamometers often are used to assess hand function in children. The use of normative grip strength data at followup is difficult because of the influence of growth and neuromuscular maturation. As an alternative, infant welfare centers throughout the world use growth diagrams to observe normative growth. The aim of this study was to develop similar growth diagrams for grip strength in children. We measured the grip strength, hand
\end{abstract}

One or more of the authors (HMM, RWS) have received funding from Fonds Nuts Ohra (http://www.fondsnutsohra.nl), a nonprofit foundation supporting research with no professional interest in the design and outcome of this study.

Each author certifies that his or her institution has approved the human protocol for this investigation, that all investigations were conducted in conformity with ethical principles of research, and that informed consent for participation in the study was obtained.

H. M. Molenaar $(\bowtie)$, R. W. Selles

Department of Rehabilitation, Erasmus MC, University Medical Center, Rotterdam, The Netherlands

e-mail: H.Molenaar@erasmusmc.nl

H. M. Molenaar, R. W. Selles

Department of Plastic and Reconstructive Surgery, Erasmus MC, Room Ee1591, Dr Molewaterplein 50, 3015GD Rotterdam, The Netherlands

J. M. Zuidam, S. E. R. Hovius

Department of Plastic and Reconstructive Surgery, Erasmus MC, University Medical Center, Rotterdam, The Netherlands

\section{S. P. Willemsen}

Department of Biomedical Statistics, Erasmus MC, University Medical Center, Rotterdam, The Netherlands

H. J. Stam

Department of Rehabilitation, Erasmus MC, University Medical Center, Rotterdam, The Netherlands dominance, gender, height, and weight of 225 children, 4 to 12 years old. We developed separate statistical models for both hands of boys and girls for drawing growth curves. Grip strength increased with age for both hands. For the whole group, the dominant hand produced higher grip strength than the nondominant hand and boys were stronger than girls. The grip strength of boys and girls differed between 2 and $19 \mathrm{~N}$ for the different age groups. Because grip strength measurements are accompanied by a rather large variance, the growth diagrams (presenting a continuum in grip strength) make it possible to better observe grip strength development with time corresponding to a more exact age. Depending on the accuracy needed, the use of one combined diagram could be considered.

Level of Evidence: Level II, diagnostic study. See Guidelines for Authors for a complete description of levels of evidence.

\section{Introduction}

Grip strength measurements often are used to assess hand function in patients with trauma or congenital problems or in the case of degenerative diseases [12]. Hand dynamometers frequently used to measure grip strength are the Jamar dynamometer (TEC, Clifton, NJ) or equivalent devices such as the Lode dynamometer (Lode BV, Groningen, The Netherlands).

Normative and reliability data on these dynamometers have been published for adults and children [3-5, 14-20]. For adults, a reference table with normative data generally is used. For children, normative data often are presented in a table format, in which mean grip strength data are given for 1- or 2-year intervals with standard deviations (SDs) $[8,16]$. Alternatively, studies have presented equations 
relating grip strength data with variables such as age, gender, height, weight, or body mass index [20].

Using the normative data as reference values can be cumbersome when measuring a child at followup owing to the increase in strength when a child grows. A child not only increases in length but also in weight, bone mass, and muscle volume. This growth complicates the interpretation of changes in strength measured after interventions such as surgery or rehabilitation, because outcome is influenced by growth and the intervention. To discriminate among contributions of growth, intervention effects, or disease progression, a model more easily interpreted than a table with 1 - or 2-year intervals would be valuable.

As an alternative to the mentioned presentation of normative data, in children, an intuitive diagram in which strength is plotted against age would provide an immediate indication of the strength that can be expected at the child's age using a continuous age scale. In addition, variation in strength can be accounted for using the correct percentiles. Such growth diagrams for length and weight have been developed for use at infant welfare centers across the world $[6,7,10,11]$. Use of these diagrams for grip strength would give a quick and easy insight into grip strength development during growth. For example, it could be very valuable when treating children with congenital hand malformations or children with neuromuscular disorders who receive longterm treatment to follow the child's progress.

Our first aim was to create growth diagrams for grip strength, making normative data of grip strength more intuitive and easily accessible from a clinical point of view. To do so, we first determined which variables in addition to age, such as hand dominance, gender, height, and weight, influenced grip strength in our population. Then, we used these variables to develop a model for drawing grip strength growth curves in an easily interpretable diagram for clinical use. The second aim of our study was to see if an accurate model for grip strength still could be developed using only simplified average analysis combining both genders and dominant hand and nondominant hands into one diagram.

\section{Materials and Methods}

After approval of the Institutional Review Board and after informed consent of the parents, we approached children from a local primary school without upper limb impairment for participation. Hand dominance and upper extremity problems that could influence hand strength were determined by evaluating parents' responses to a questionnaire. Children with a history of upper extremity trauma or abnormalities were excluded. For this study, we measured an extra 121 children in addition to the 104 children used in a previous study of the reliability of the grip strength dynamometer in children [17]. Two hundred twenty-five children, aged 4 to 12 years, were included in this study. Of all the children studied, 94\% were right-handed (Table 1).

We used a Jamar-like dynamometer (Lode dynamometer) for all measurements (Fig. 1). The Lode dynamometer is an electronic dynamometer similar to the Jamar dynamometer. It operates similarly and is calibrated to measure the same outcome as the Jamar dynamometer. In a previous study, we quantified the measurement error of this instrument in children and found the Lode dynamometer to be reliable in healthy children from 4 to 12 years old. Reliability increased with age and children of 12 years old had similar reliability as adults [17]. The dynamometer was used as recommended by the American Society of Hand Therapists [2]. The subject sits with the shoulder adducted, the elbow flexed in a $90^{\circ}$ angle, and the wrist in a neutral position [2].

The Lode dynamometer, with the handlebar in Position 2, was used to measure grip strength of both hands [9]. After each measurement, we reset the dynamometer. All measurements were performed in a randomized order by the same researcher (HMM). The children were seated in an appropriately adjusted chair during measurements. Before the start of each measurement, the subject was told, "Squeeze as hard as you can!" A mean of three maximum voluntary contractions was recorded for each hand. In case one of the measurements showed a difference greater than $10 \%$ from the other measurements, we cancelled that measurement and added a fourth measurement. The mean of the three remaining values was calculated.

To develop the growth curves, we first estimated the centiles for grip strength using Altman's method of absolute scaled residuals [1]. Because a visual inspection did not reveal skewness or nonnormal kurtosis, we decided not to transform the dependent variable. In the first model, grip strength was modeled as a function of the age. To allow for nonlinearity in the mean, we used restricted cubic splines with three knots placed at the 10th, 50th, and 90th centiles.

Table 1. Number of participants divided by gender and age

\begin{tabular}{lccc}
\hline Age (years) & Number of boys & Number of girls & Total \\
\hline 4 & 12 & 11 & 23 \\
5 & 13 & 13 & 26 \\
6 & 11 & 12 & 23 \\
7 & 13 & 14 & 27 \\
8 & 12 & 15 & 27 \\
9 & 12 & 14 & 26 \\
10 & 13 & 12 & 25 \\
11 & 12 & 12 & 24 \\
12 & 12 & 12 & 24 \\
Total & 110 & 115 & 225 \\
\hline
\end{tabular}




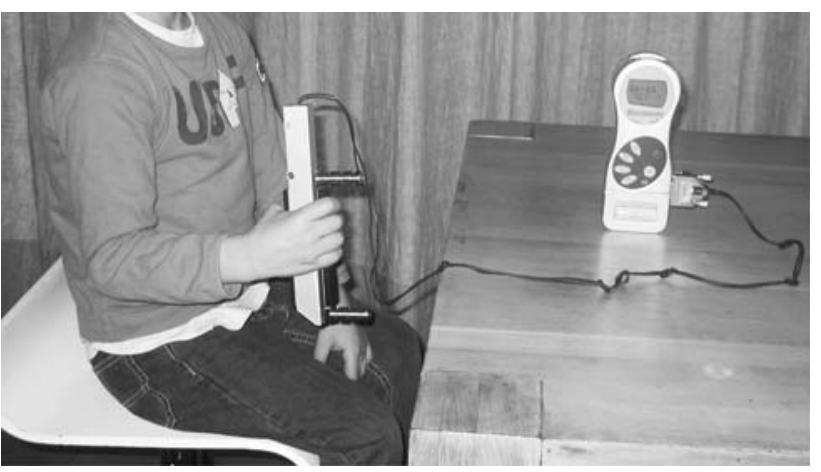

Fig. 1 A 6-year-old child holds a Jamar-like dynamometer (Lode dynamometer). The electronic console was facing the researcher so that no feedback was provided to the subjects.

The SD was estimated using the regression function of the absolute residuals. We estimated separate relationships for boys and girls for the dominant and nondominant hands.

The mentioned model was compared with a more complex model that also included weight and length next to age. All factors were included in this more complex model as a restricted cubic spline with three knots. Residuals of the various models were checked for normality and serial correlation.

We used a partial $\mathrm{F}$ test to calculate differences in grip strength by gender and hand dominance. Although we found major differences between boys and girls and between both hands, we also made a combined diagram for all boys, girls, and both hands combined. Although this combined graph is less accurate in predicting normative data for an individual subject, we present it for use as a first estimate in clinical situations in which four separate graphs may be too cumbersome. All estimations and calculations were performed using SAS ${ }^{\circledR} 9.1$ (SAS Institute, Inc, Cary, NC).

\section{Results}

Grip strength increased with age in both hands. In the entire group, the dominant hand produced higher $(\mathrm{p}<0.001)$ grip strength than the nondominant hand and boys were stronger $(\mathrm{p}<0.001)$ than girls (Tables 2,3$)$. The differences between boys and girls were not the same for each age. For the youngest group of 4 to 6 years, the boys were $24 \%$ to $34 \%$ stronger; for the middle group of 7 to 9 years old, the boys were $2 \%$ to $9 \%$ stronger; and for the oldest group of 10 to 12 years, the boys were $3 \%$ to $11 \%$ stronger. For both genders, differences in grip strength between dominant and nondominant hands ranged from 2 to $17 \mathrm{~N}$. To determine correct models for predicting grip strength in the dominant and nondominant hands of the boys and girls separately, we compared the more complex
Table 2. Grip strength for boys

\begin{tabular}{|c|c|c|c|c|c|c|}
\hline \multirow[t]{2}{*}{ Hand } & \multirow{2}{*}{$\begin{array}{l}\text { Age } \\
\text { (years) }\end{array}$} & \multirow{2}{*}{$\begin{array}{l}\text { Number } \\
\text { of boys }\end{array}$} & \multicolumn{4}{|c|}{ Grip strength $(\mathrm{N})^{*}$} \\
\hline & & & Mean & SD & Minimum & Maximum \\
\hline Dominant & 4 & 12 & 65.9 & 12.9 & 50.2 & 93.1 \\
\hline Nondominant & & & 61.7 & 14.2 & 39.9 & 85.2 \\
\hline Dominant & 5 & 13 & 84.0 & 17.6 & 53.3 & 112.8 \\
\hline Nondominant & & & 73.5 & 14.4 & 47.3 & 94.8 \\
\hline Dominant & 6 & 11 & 97.6 & 16.3 & 70.0 & 115.4 \\
\hline Nondominant & & & 92.2 & 16.7 & 64.7 & 123.9 \\
\hline Dominant & 7 & 13 & 115.7 & 22.0 & 80.2 & 145.7 \\
\hline Nondominant & & & 106.1 & 15.6 & 83.9 & 135.6 \\
\hline Dominant & 8 & 12 & 115.8 & 29.2 & 69.3 & 152.9 \\
\hline Nondominant & & & 110.8 & 27.4 & 69.8 & 158.0 \\
\hline Dominant & 9 & 12 & 139.6 & 24.0 & 108.2 & 179.8 \\
\hline Nondominant & & & 137.1 & 24.0 & 102.9 & 176.7 \\
\hline Dominant & 10 & 13 & 159.5 & 36.3 & 126.9 & 266.1 \\
\hline Nondominant & & & 151.9 & 37.4 & 96.4 & 245.9 \\
\hline Dominant & 11 & 12 & 195.4 & 36.0 & 139.9 & 251.7 \\
\hline Nondominant & & & 179.8 & 32.8 & 142.9 & 251.1 \\
\hline Dominant & 12 & 12 & 219.4 & 35.0 & 151.1 & 288.8 \\
\hline Nondominant & & & 202.8 & 31.8 & 147.9 & 271.2 \\
\hline
\end{tabular}

* Shown as the mean, SD, minimum, and maximum values of the maximum voluntary contraction; $\mathrm{SD}=$ standard deviation.

Table 3. Grip strength for girls

\begin{tabular}{|c|c|c|c|c|c|c|}
\hline \multirow[t]{2}{*}{ Hand } & \multirow{2}{*}{$\begin{array}{l}\text { Age } \\
\text { (years) }\end{array}$} & \multirow{2}{*}{$\begin{array}{l}\text { Number } \\
\text { of girls }\end{array}$} & \multicolumn{4}{|c|}{ Grip strength $(\mathrm{N})^{*}$} \\
\hline & & & Mean & SD & Minimum & Maximum \\
\hline Dominant & 4 & 11 & 48.6 & 12.7 & 36.2 & 70.8 \\
\hline Nondominant & & & 46.3 & 11.9 & 25.3 & 61.0 \\
\hline Dominant & 5 & 13 & 64.1 & 16.9 & 44.3 & 97.2 \\
\hline Nondominant & & & 59.3 & 14.1 & 31.4 & 79.1 \\
\hline Dominant & 6 & 12 & 82.7 & 19.1 & 57.9 & 123.5 \\
\hline Nondominant & & & 70.5 & 10.2 & 53.8 & 87.4 \\
\hline Dominant & 7 & 14 & 107.4 & 18.1 & 86.0 & 140.6 \\
\hline Nondominant & & & 96.9 & 15.1 & 78.7 & 130.8 \\
\hline Dominant & 8 & 15 & 116.0 & 23.0 & 77.4 & 148.4 \\
\hline Nondominant & & & 107.1 & 19.0 & 73.2 & 136.2 \\
\hline Dominant & 9 & 14 & 133.8 & 29.9 & 93.7 & 185.2 \\
\hline Nondominant & & & 126.3 & 27.7 & 83.7 & 176.3 \\
\hline Dominant & 10 & 12 & 152.2 & 32.6 & 83.3 & 193.0 \\
\hline Nondominant & & & 138.6 & 33.6 & 68.1 & 174.9 \\
\hline Dominant & 11 & 12 & 190.2 & 32.5 & 151.0 & 249.3 \\
\hline Nondominant & & & 175.0 & 30.5 & 137.8 & 231.7 \\
\hline Dominant & 12 & 12 & 197.6 & 42.6 & 109.2 & 265.4 \\
\hline Nondominant & & & 181.9 & 34.8 & 105.4 & 223.3 \\
\hline
\end{tabular}

* Shown as the mean, SD, minimum, and maximum values of the maximum voluntary contraction; $\mathrm{SD}=$ standard deviation. 
Table 4. $\mathrm{R}^{2}$ of the models used to draw growth curves for boys and girls separately and for both genders combined

\begin{tabular}{lll}
\hline Gender, hand dominance & Model variable* & $\mathrm{R}^{2 \dagger}$ \\
\hline Boys, dominant & Age & $79 \%$ \\
Boys, dominant & Age, height, weight & $80 \%$ \\
Girls, dominant & Age & $80 \%$ \\
Girls, dominant & Age, height, weight & $83 \%$ \\
Boys, nondominant & Age & $77 \%$ \\
Boys, nondominant & Age, height, weight & $79 \%$ \\
Girls, nondominant & Age & $80 \%$ \\
Girls, nondominant & Age, height, weight & $84 \%$ \\
All combined (both hands, both genders) & Age & $76 \%$ \\
All combined (both hands, both genders) & Age, height, weight & $80 \%$ \\
\hline
\end{tabular}

* The simpler model accounts for age only whereas the more complex model accounts for height and weight along with age; ${ }^{\dagger} \mathrm{R}^{2}$ indicates the explained variance of the simple model, including age, and the more complex model, including age, height, and weight.

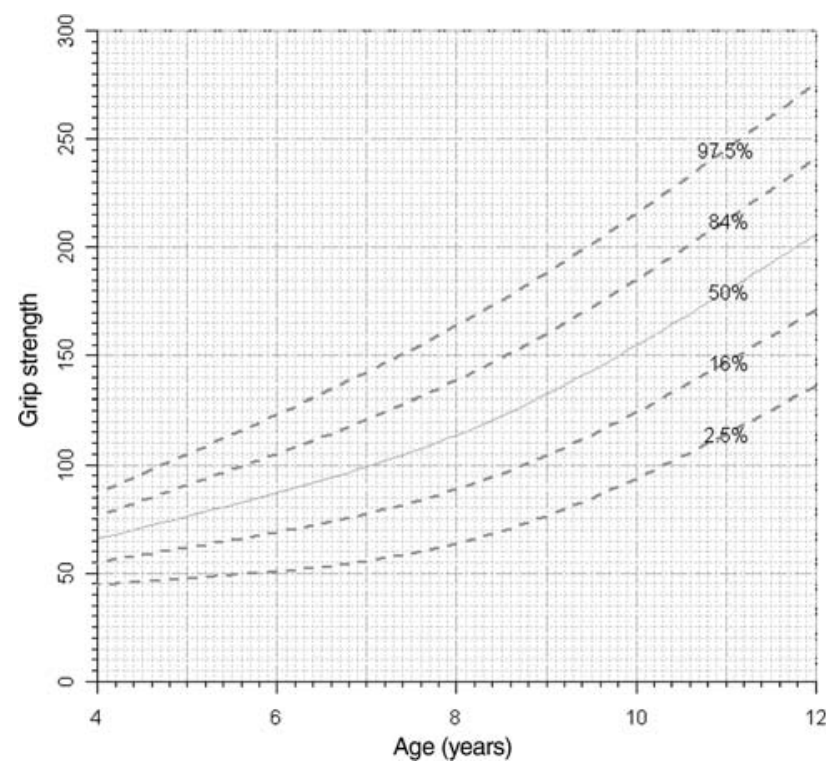

Fig. 2 A graph shows grip strength for the dominant hand in boys plotted against age. The centiles $2.5 \%, 16 \%, 50 \%, 84 \%$, and $97.5 \%$ are shown.

model including the variables age, height, weight, and gender with the simpler model that included age alone. $\mathrm{R}^{2}$ for the dominant and nondominant hands were only slightly lower in the simpler model with age as the only independent variable of grip strength (Table 4). Because we decided the benefits of a simpler model using age only outweigh the slight increase in predictability of grip strength achieved by the more complex model, we present only the simpler model. The simpler statistical models for grip strength as a function of age in the dominant and nondominant hands of boys and girls separately were converted into growth diagrams (Figs. 2-5). In addition to the curve of the population mean, these diagrams show the

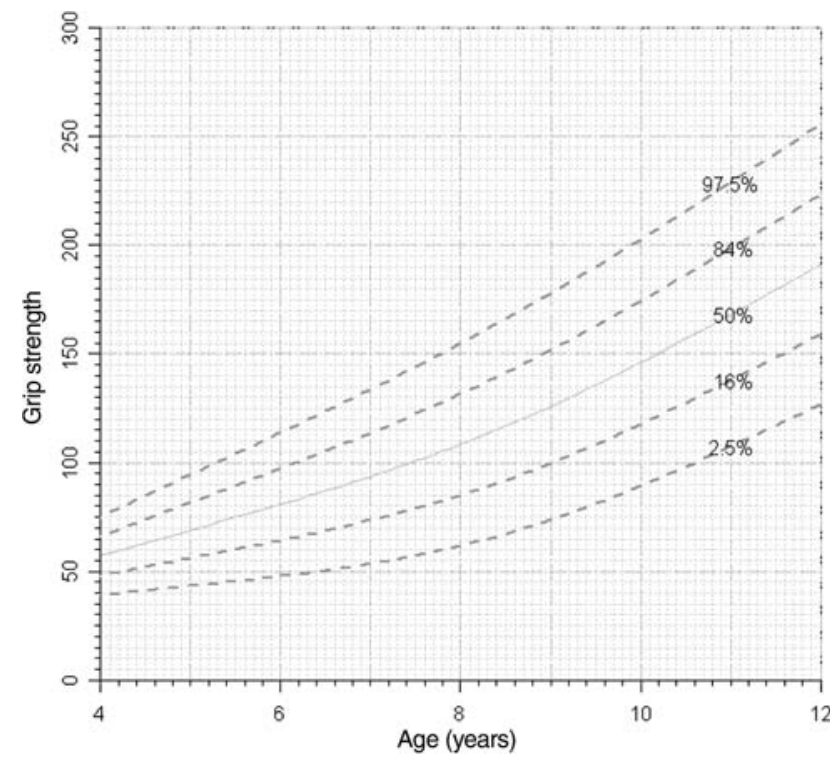

Fig. 3 A graph shows grip strength for the nondominant hand in boys plotted against age. The centiles $2.5 \%, 16 \%, 50 \%, 84 \%$, and $97.5 \%$ are shown.

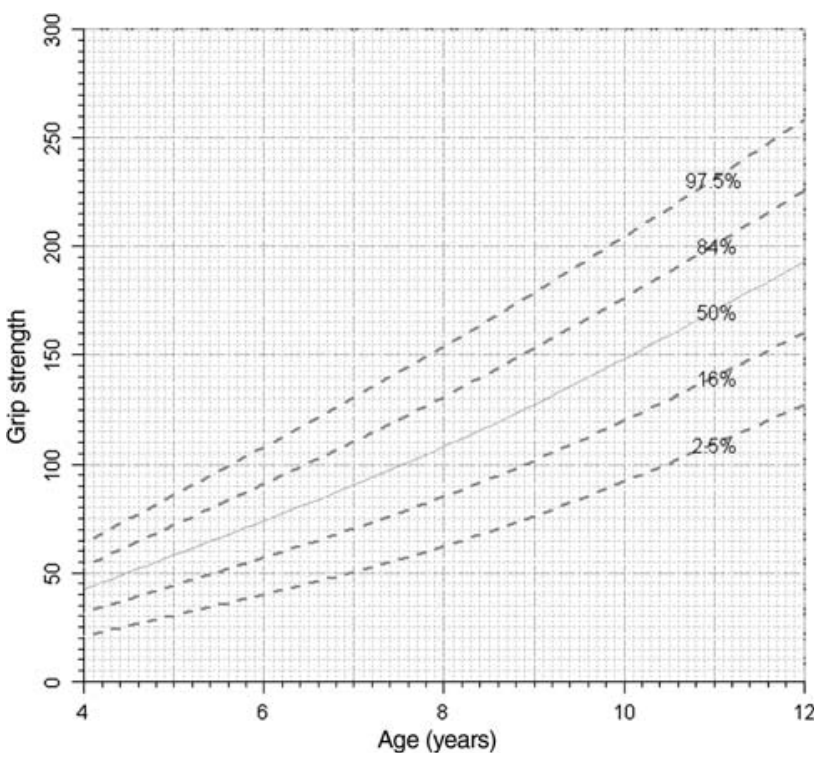

Fig. 4 A graph shows grip strength for the dominant hand in girls plotted against age. The centiles $2.5 \%, 16 \%, 50 \%, 84 \%$, and $97.5 \%$ are shown.

centiles that correspond to each standard deviation (SD) added or subtracted from the mean: $2.5 \%, 16 \%, 50 \%, 84 \%$, and $97.5 \%$ centiles correspond to $-2 \mathrm{SD},-1 \mathrm{SD}$, mean, $+1 \mathrm{SD}$, and $+2 \mathrm{SD}$.

Finally, we plotted the mean hand strength of the dominant and nondominant hands against age for boys and girls combined (Fig. 6). The use of just one diagram for both genders and both hands could be considered more practical in some clinical situations where four different diagrams are too inconvenient to use. Because this 


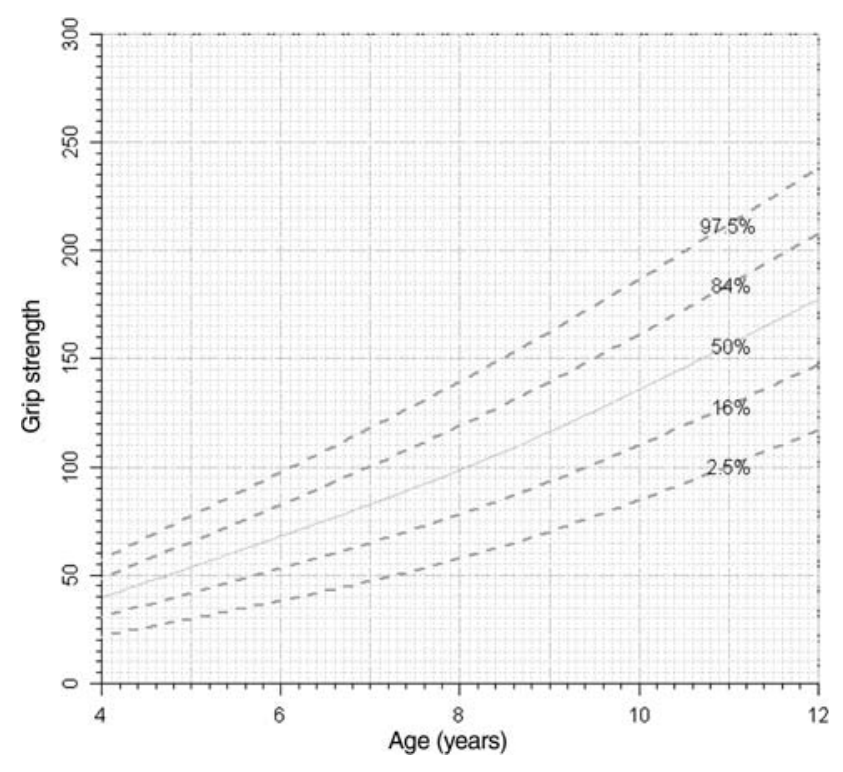

Fig. 5 A graph shows grip strength for the nondominant hand in girls plotted against age. The centiles $2.5 \%, 16 \%, 50 \%, 84 \%$, and $97.5 \%$ are shown.

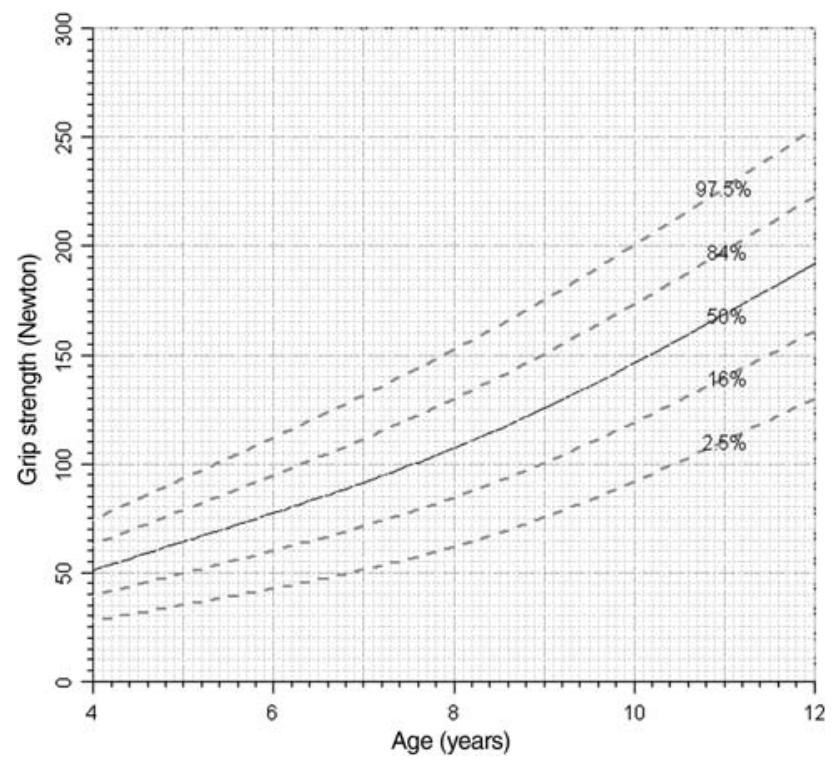

Fig. 6 A graph shows grip strength for the mean hand strength (dominant and nondominant combined) for all subjects (boys and girls combined) plotted against age. The centiles $2.5 \%, 16 \%, 50 \%, 84 \%$, and $97.5 \%$ are shown.

combined diagram includes all data not selective for gender and hand dominance, the goodness of fit is lower compared with the other diagrams (Figs. 2-5), although this effect on the goodness of fit is only small (Table 4).

\section{Discussion}

Our general aim in this study was to develop a simple and intuitive method of presenting normative grip strength data in children. Normative data usually are reported in table format, presenting grip strength by age group, gender, and hand dominance. However, when this method is used to compare grip strength values in children with time, it becomes difficult to verify grip strength changes with time against the reference data. The wide ranges in grip strength per age do not give a clear overview of a child's grip strength development with time.

As an alternative to the classic method of reference tables, we developed an intuitive diagram showing grip strength development with time, the intention being to allow for easier and insightful registration of a child's grip strength development in the same way as growth diagrams are developed for increase in height with time. We developed the growth diagrams for grip strength (Figs. 2-5) as tools to be used in a clinical setting. By allowing a child's grip strength to be plotted with time, they show how his or her grip strength has developed relative to the reference data contained in the diagram. If a child's strength is plotted at a certain distance from a centile line, a change in this distance at followup may indicate an increase or decrease in strength relative to his or her age. An additional advantage for individual patient measurements is the continuous nature of the diagrams. A table containing reference data is difficult to use, as such values are given per year or at 2-year intervals. In contrast, the growth diagrams present a continuum in grip strength values, allowing measurement outcome to be compared with a more exact age of any child.

In addition, we also present a model for both hands and with boys and girls combined (Fig. 6). By sacrificing some goodness of fit (Table 4), the use of just one diagram could be more practical in clinical settings. One important thing to consider using these models (Figs. 2-6) is that the centile lines are based on the SD of these measurements. As a result, $5 \%$ of all healthy children automatically will fall outside the outer centile lines.

The grip strength data in our study were somewhat lower than the reported normative data for grip strength measured using a handheld dynamometer. To prevent inadvertent dropping, Mathiowetz et al. [16] supported the instrument around the readout dial, and they scored a higher grip strength of $30 \%$ or greater. In another study, van den Beld et al. [20] used a height-adjustable table on which to rest the instrument. Their results in grip strength were approximately $19 \%$ higher [20]. Finally, de Smet and Vercammen [8] used no suspension method and measured grip strengths that were approximately 5\% higher. The lower grip strength in our study may partly be explained by the fact that we used no form of suspension and that the children therefore needed to lift the weight of the instrument. We recently found suspension of the dynamometer when measuring grip in similar age groups can increase the 
force output by $10 \%$. We also found the reliability did not increase by adding visual feedback to the strength measurements (Unpublished study: Molenaar HM, Selles RW, M Kd, Zuidam JM, Stam HJ, Hovius SE. Reliability of three different protocols for measuring grip strength in children. EUROHAND 2008 - XIIIth Congress of the FESSH - IXth Congress of the EFSHT. Lausanne, Switzerland; 2008).

Some limitations must be mentioned regarding our study. First, the growth diagrams apply only to a healthy population of children and do not represent the development of the grip strength of a child with hand disorders such as neuromuscular diseases or congenital malformations. Furthermore, our data apply only to the age range of 4 to 12 years and no reliable predictions can be made for children older than 12 years. In a previous study, we quantified the measurement error of this instrument in children. The Lode dynamometer was found to be reliable in healthy children from 4 to 12 years old. Reliability increased with age and children 12 years old had reliability similar to that of adults [17]. In addition, based on our clinical experience when measuring children, it is generally too difficult to reliably measure children as young as 3 years. For these reasons, we used the lower age limit of 4 years. We studied the group from 4 to 12 years old, as this age group is relevant in our work with patients with congenital hand malformations. During the period from 4 to 12 years, these children often are treated multiple times for their specific congenital malformations. Intervention and intensive therapy are very important to their development, dexterity, and growth.

Another limitation of our study is that the diagrams summarize cross-sectional grip strength data and not longitudinal data taken from a group of children measured repeatedly during their development. In addition, there is a large variance in measurement outcome when repeated grip strength measurements are performed in reliability studies $[13,15,19,20]$. A quantification of variance in a recent paper regarding the reliability of the hand strength dynamometer in children showed a smallest detectable difference of $23 \%$ to $27 \%$ of the outcome [17]. Adding more subjects to our study will not change the distance between the centile lines in the model; it would only allow for a more accurate estimation of the mean and centiles lines.

In clinical settings in which it is important to follow the development of a child's grip strength, a growth diagram would give a good indication of individual development relative to normative values. In this way, a possible effect of treatment or therapy may be seen quickly as a gain or loss of grip strength with time. Our grip strength plots (Figs. 2-5) for each specific target group provide the observer with the best model for comparing measurement outcome. Because these diagrams are more specific, they show a better fit to the data. Depending on the accuracy needed at followup, the use of one diagram might be considered. At the cost of losing some goodness of fit, one combined diagram would be much simpler and more convenient to use (Fig. 6). Either way, because the diagrams give observers a practical tool for tracking multiple grip strength measurements of a growing child with time, they might be suitable for inclusion in a patient dossier.

Acknowledgments We thank Eric Teunisse, director of the Montessori School in Kralingen (MSK; Rotterdam, The Netherlands) for his cooperation during our measurements. In addition, we thank all the teachers and children of the MSK.

Open Access This article is distributed under the terms of the Creative Commons Attribution Noncommercial License which permits any noncommercial use, distribution, and reproduction in any medium, provided the original author(s) and source are credited.

\section{References}

1. Altman DG. Construction of age-related reference centiles using absolute residuals. Stat Med. 1993;12:917-924.

2. American Society of Hand Therapists. Clinical Assessment Recommendations. Ed 2. Chigago, IL; 1992.

3. Bellace JV, Healy D, Besser MP, Byron T, Hohman L. Validity of the Dexter Evaluation System's Jamar dynamometer attachment for assessment of hand grip strength in a normal population. $J$ Hand Ther. 2000;13:46-51.

4. Bohannon RW, Schaubert KL. Test-retest reliability of gripstrength measures obtained over a 12-week interval from community-dwelling elders. $J$ Hand Ther. 2005;18:426-427, quiz 428.

5. Clerke AM, Clerke JP, Adams RD. Effects of hand shape on maximal isometric grip strength and its reliability in teenagers. $J$ Hand Ther. 2005;18:19-29.

6. Cole TJ. Using the LMS method to measure skewness in the NCHS and Dutch National height standards. Ann Hum Biol. 1989;16:407-419.

7. Cole TJ. The LMS method for constructing normalized growth standards. Eur J Clin Nutr. 1990;44:45-60.

8. De Smet L, Vercammen A. Grip strength in children. J Pediatr Orthop B. 2001;10:352-354.

9. Firrell JC, Crain GM. Which setting of the dynamometer provides maximal grip strength? J Hand Surg Am. 1996;21:397-401.

10. Fredriks AM, van Buuren S, Jeurissen SE, Dekker FW, VerlooveVanhorick SP, Wit JM. Height, weight, body mass index and pubertal development reference values for children of Turkish origin in the Netherlands. Eur J Pediatr. 2003;162:788-793.

11. Fredriks AM, van Buuren S, Jeurissen SE, Dekker FW, VerlooveVanhorick SP, Wit JM. Height, weight, body mass index and pubertal development references for children of Moroccan origin in The Netherlands. Acta Paediatr. 2004;93:817-824.

12. Gupta A, Kay S, Scheker R. The Growing Hand: Diagnosis and Management of the Upper Extremity in Children. London, England: Mosby; 2000.

13. Mathiowetz V. Comparison of Rolyan and Jamar dynamometers for measuring grip strength. Occup Ther Int. 2002;9:201-209.

14. Mathiowetz V, Kashman N, Volland G, Weber K, Dowe M, Rogers S. Grip and pinch strength: normative data for adults. Arch Phys Med Rehabil. 1985;66:69-74. 
15. Mathiowetz V, Weber K, Volland G, Kashman N. Reliability and validity of grip and pinch strength evaluations. J Hand Surg Am. 1984;9:222-226.

16. Mathiowetz V, Wiemer DM, Federman SM. Grip and pinch strength: norms for 6- to 19-year-olds. Am J Occup Ther. 1986;40:705-711.

17. Molenaar HM, Zuidam JM, Selles RW, Stam HJ, Hovius SE. Age-specific reliability of two grip-strength dynamometers when used by children. J Bone Joint Surg Am. 2008;90:1053-1059.

18. Schreuders TA, Roebroeck ME, Goumans J, van Nieuwenhuijzen JF, Stijnen TH, Stam HJ. Measurement error in grip and pinch force measurements in patients with hand injuries. Phys Ther. 2003;83:806-815.

19. Smidt N, van der Windt DA, Assendelft WJ, Mourits AJ, Deville WL, de Winter AF, Bouter LM. Interobserver reproducibility of the assessment of severity of complaints, grip strength, and pressure pain threshold in patients with lateral epicondylitis. Arch Phys Med Rehabil. 2002;83:1145-1150.

20. van den Beld WA, van der Sanden GA, Sengers RC, Verbeek AL, Gabreels FJ. Validity and reproducibility of the Jamar dynamometer in children aged 4-11 years. Disabil Rehabil. 2006; 28:1303-1309. 